\title{
ACTIVIDAD EMULSIFICANTE Y DE REMOCIÓN DE METALES PESADOS DEL RAMNOLÍPIDO PRODUCIDO POR Pseudomonas aeruginosa PB 25
}

\author{
J.Daniel Giraldo ${ }^{\mathrm{a}}$, Susana Gutiérrez ${ }^{\mathrm{a}^{*}}$ y Fernando Merino ${ }^{\mathrm{a}}$
}

\section{RESUMEN}

El avance científico-tecnológico realizado desde la revolución industrial, ha aumentado la capacidad del ser humano para explotar los recursos naturales causando una constante perturbación en los ecosistemas. En este contexto, el uso de los biosurfactantes, representa una prometedora alternativa de aplicación para procesos de remediación de ambientes naturales. El objetivo del presente trabajo fue evaluar la actividad emulsificante y de remoción de metales pesados de un biosurfactante de naturaleza ramnolipídica producido por Pseudomonas aeruginosa PB25. Esta creció con una velocidad específica $(\mu)$ de $0,0285 \mathrm{~h}^{-1} \mathrm{y}$ un tiempo generacional $\left(\mathrm{t}_{\mathrm{g}}\right)$ de $24,321 \mathrm{~h}$; registrándose a su vez una concentración máxima de $2,47 \mathrm{~g} / \mathrm{L}$ de ramnolípidos en la fase estacionaria de crecimiento, con valores de rendimiento (Y) de 0,13 gramos de ramnolípido por gramo de glicerol y de productividad de 0,082 g/L-h. El ramnolípido alcanzó 5,257 Unidades de Actividad Emulsificante $/ \mathrm{mL}$ frente a crudo de petróleo e índices de emulsificación E24 de 53, 64, 62 y 84 \% para crudo de petróleo, petróleo diesel 2, gasolina y kerosene, respectivamente. Logró remover $98 \%$ de plomo y $99 \%$ de cadmio en soluciones acuosas a pH 11. Por lo cual, este biosurfactante puede ser empleado en procesos de biorremediación. .

Palabras clave: Remediación, biosurfactantes, ramnolípidos.

\section{OIL EMULSIFYING ACTIVITY AND REMOVAL OF HEAVY METALS BY PSEUDOMONAS AERUGINOSA PB 25 RHAMNOLIPID}

\begin{abstract}
Since the industrial revolution, the science and technology advances have increased the human ability to exploit natural resources causing pollution in ecosystems. In this context, the use of biosurfactants represents a promising alternative application for any technological process of remediation of natural environments.

The objective of this work was the evaluation of the emulsifying activity and the ability to remove heavy metals with a rhamnolipidic biosurfactant produced by Pseudomonas aeruginosa PB25. This strain had a specific growth rate $(\mu)$ of $0.0285 \mathrm{~h}^{-1}$ and a generational time (td) of $24.321 \mathrm{~h}$. It produced $2.47 \mathrm{~g} / \mathrm{L}$ rhamnolipid, with yields (Y) of $0.13 \mathrm{~g} / \mathrm{g}$ and productivity of $0.082 \mathrm{~g} / \mathrm{L}-\mathrm{h}$. The rhamnolipid had 5.257 emulsifying activity units $/ \mathrm{mL}$ and E24 emulsification index of 53, 64, 62 and 84\% for crude oil, diesel oil 2, gasoline and kerosene, respectively. It got to remove $98 \%$ of lead and $99 \%$ of cadmium in aqueous solutions at $\mathrm{pH} 11$.

In conclusion, it can be used in biotechnological processes.
\end{abstract}

Key words: Remediation, biosurfactants, rhamnolipids.

\footnotetext{
${ }^{a^{*}}$ Facultad de Ciencias Biológicas, Universidad Nacional Mayor de San Marcos, Av. Venezuela Cdra 34 s/n, Lima 1, Perú. sugutimer@gmail.com.
} 


\section{INTRODUCCIÓN}

El tratamiento de zonas contaminadas por metales pesados o hidrocarburos de petróleo requiere, generalmente, del uso de sustancias sintéticas que si bien pueden ser altamente efectivas, poseen también un grado de toxicidad, llegando incluso a ser destructivas para la estructura física, química o biológica de los suelos o aguas ${ }^{1,2}$, por lo que su uso no es el más adecuado en situaciones donde el objetivo principal de un proceso de remediación es retornar la zona afectada a un estado saludable y productivo. El uso de sustancias de origen biológico ofrece una nueva alternativa de solución amigable con el medio ambiente, especialmente en la ausencia de tecnologías que contribuyan a solucionar eficazmente los problemas ocasionados por las actividades industriales del hombre; más aun, tomando en cuenta que en nuestro país no existe o son muy pocas las referencias acerca del uso de ese tipo particular de sustancias.

Los ramnolípidos han sido ampliamente estudiados y cerca de 7 homólogos han sido identificados hasta el momento ${ }^{3}$. Tensiones superficiales de $29 \mathrm{mN} / \mathrm{m}$ ( mili Newtons/ metro) son características de este tipo de compuestos que pueden contener dos moléculas de ramnosa unidas al ácido $\alpha$-hidroxidecanoico (di-ramnolípidos) o una sola molécula de ramnosa unida a un ácido graso idéntico (mono-ramnolípidos) ( figura 1) .

Dentro de la variedad de microorganismos productores de estas sustancias, los que mayor potencial aplicativo engloban son las especies pertenecientes al género Pseudomonas, las cuales típicamente producen biosurfactantes de naturaleza glicolipídica llamados ramnolípidos ${ }^{4,5,6}$.

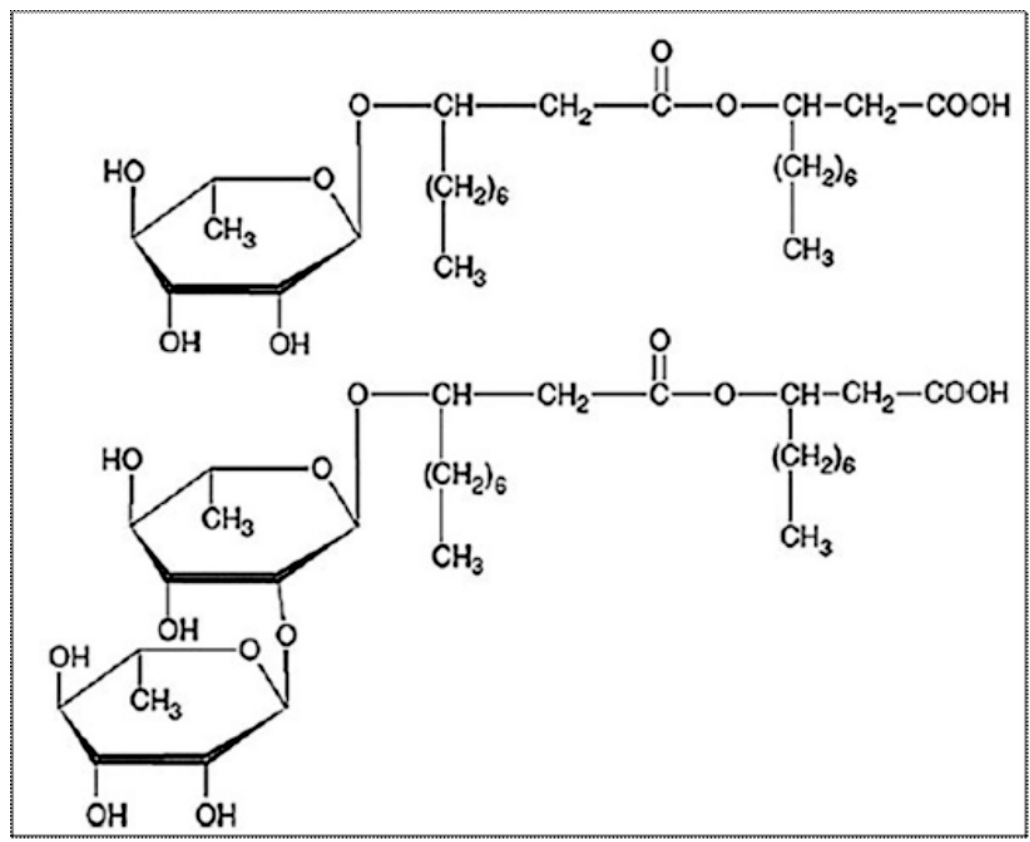

Figura 1.: Representación molecular de mono-ramnolípidos (parte superior) y diramnolípidos (parte inferior) producidos por Pseudomonas aeruginosa. Fuente: Lovaglio et al., $2011^{7}$. 
Los ramnolípidos producidos por Pseudomonas aeruginosa pueden obtenerse a partir de diversos sustratos de bajo costo que van desde azúcares hasta hidrocarburos y pueden incluir compuestos tales como alcanos, piruvatos, citratos, fructosa, aceites vegetales, glicerol, glucosa, manitol e incluso mediante el uso de residuos agroindustriales o aceites vegetales reciclados usados como fuentes de carbono ${ }^{8,9,10,11}$.

Dentro de las múltiples aplicaciones que se atribuye a los ramnolípidos, una de las más importantes es su potencial uso en procesos de biorremediación ${ }^{4}$; también se indica que la adición de ramnolípidos puede incrementar la biodegradación de n-parafinas y fenantreno en sistemas líquidos y mezclas de hidrocarburos en suelos ${ }^{12,13}$. Este hecho es de gran importancia para la estimulación de la degradación bacteriana bajo condiciones in situ.

El uso de surfactantes ramnolípidos fue capaz de liberar hasta cerca de 3 veces tanto crudo como el agua sola de las playas de Alaska luego del derrame del Exxon Valdez ${ }^{14,15}$.

Por otra parte, su habilidad de unión de los ramnolípidos a iones metálicos específicos, facilita su empleo en procesos de extracción mejorada de metales, tales como el cobre, a partir de actividades mineras o la remoción de metales pesados, tales como el plomo o el cadmio, presentes como contaminantes en suelos y aguas ${ }^{16,17,1,18}$.

También cabe resaltar que debido a su compatibilidad con la piel y su bajo poder irritante, los ramnolípidos son usados actualmente como aditivos en la industria cosmética por la empresa Iwata Co. de Japón ${ }^{12,19}$ que posee patentes para el uso de ramnolípidos en liposomas y emulsiones para la industria cosmética.

Finalmente, es sumamente importante resaltar que, debido al uso exitoso de cualquier compuesto en un proceso tecnológico, requiere necesariamente del conocimiento previo de las características propias del mismo ${ }^{20}$. Tomando en cuenta que no existe referencia en nuestro país acerca de la producción y empleo de ramnolípidos o de algún otro tipo de biosurfactante; este trabajo de investigación se enfocó, principalmente, en la evaluación de dos de las características claves de este tipo de compuestos (actividad emulsificante y capacidad de remoción de metales pesados), estudio preliminar necesario para su posterior empleo en procesos a mayor escala de biorremediación de ambientes naturales contaminados con metales pesados y/o hidrocarburos.

\section{PARTE EXPERIMENTAL}

\section{Selección de la cepa bacteriana:}

La cepa bacteriana se escogió del banco de cepas del Laboratorio de Microbiología y Biotecnología Microbiana de la Facultad de Ciencias Biológicas de la UNMSM, que contiene cepas aisladas de diversas zonas del Perú, contaminadas con petróleo. Se seleccionó al azar las cepas de Pseudomonas fluorescens MP, Pseudomonas aeruginosa 66, 25, 74 y AP.

La actividad degradativa de hidrocarburos se comprobó mediante el cultivo de las cepas en el medio mineral según Mills suplementado con crudo de petróleo como única fuente de carbono, incubándose a $25^{\circ} \mathrm{C}$ en condiciones estáticas y a $\mathrm{pH} 7,0$ por 5 días. Luego, se procedió a evaluar la actividad emulsificante de cada una de ellas como criterio de selección para la cepa a utilizar en el estudio, según la metodología empleada por Escalante ${ }^{21}$.

\section{Producción del biosurfactante:}

La producción, a nivel de laboratorio, del biosurfactante se llevó a cabo con el empleo del medio mínimo mineral propuesto por Zhang et al. ${ }^{6}$, suplementado con un 3\% (p/v) de glicerol como única fuente de carbono, a pH 6,8 ; incubándose a $30^{\circ} \mathrm{C}$ en condiciones estáticas durante 120 horas. 
El monitoreo del crecimiento microbiano se realizó mediante densidad óptica a $620 \mathrm{~nm}$.. El consumo de glicerol se midió con el reactivo de antrona de acuerdo al método descrito por Pons et $\mathrm{al}^{22}$. Finalmente, la concentración de ramnolípidos presentes en el medio de cultivo fue cuantificada a intervalos regulares mediante el método del orcinol según Wang et al..$^{23} \mathrm{y}$ expresadas en valores de ramnolípidos (g/L) de acuerdo a lo indicado por Zhang et al. ${ }^{6}$ y porque los microorganismos del género Pseudomonas sólo producen biosurfactantes de naturaleza ramnolipídica.

\section{Extracción y purificación de los ramnolípidos:}

La extracción de los ramnolípidos se realizó de acuerdo a la metodología descrita por Wang et $a l^{23}$ con algunas modificaciones. Para este fin, el sobrenadante del medio de cultivo fue separado del paquete celular mediante centrifugación a $5000 \mathrm{rpm}$ durante $30 \mathrm{~min}$; dicho paso fue repetido dos veces. El sobrenadante obtenido de esta manera fue acidificado hasta alcanzar un $\mathrm{pH}$ final de 2 y mantenido a $4^{\circ} \mathrm{C}$ por toda una noche. Los ramnolípidos precipitados de esta manera se recuperaron mediante centrifugación a $5000 \mathrm{rpm}$ durante 1 hora, y finalmente, disueltos en agua bidestilada. Seguidamente dos volúmenes de una mezcla de cloroformo: etanol $(2: 1, \mathrm{v} / \mathrm{v})$ se añadió a la solución de ramnolípidos, agitándose durante $30 \mathrm{~min}$. La fase orgánica obtenida de esta manera fue recolectada y puesta inmediatamente a evaporar, obteniéndose una sustancia de apariencia oleosa y color miel que al ser evaluada con el método del orcinol, es expresada como concentración de ramnolípidos ya extraídos; por ser ésta la naturaleza química correspondiente a los biosurfactantes producidos por Pseudomonas aeruginosa.

\section{Actividad emulsificante de los ramnolípidos}

La actividad emulsificante del ramnolípido fue evaluada de acuerdo a la metodología descrita por Escalante ${ }^{21}$ con algunas modificaciones, a $10 \mathrm{~mL}$ de una solución del ramnolípido al $0,1 \%$ $(\mathrm{p} / \mathrm{v})$. Se les agregó $0,2 \mathrm{~mL}$ de crudo de petróleo. Esta mezcla fue agitada manualmente durante $5 \mathrm{~min}$ para favorecer la formación de una emulsión, luego de lo cual $5 \mathrm{~mL}$ de la misma fueron transvasados a tubos de espectrofotómetro (Spectronic 20D, Milton Roy) para su lectura a $540 \mathrm{~nm}$. Finalmente, la absorvancia leída fue convertida a Unidades de Actividad Emulsificante por $\mathrm{mL}$ (UAE/mL), siendo 0,816 el equivalente de absorvancia para una Unidad de Actividad Emulsificante por $\mathrm{mL}$.

La estabilidad de las emulsiones generadas por los ramnolípidos se analizó por medio del índice de emulsificación E24, de acuerdo a la metodología descrita por Abouseoud et al. ${ }^{24}$; de este modo un volumen determinado del ramnolípido estéril $(2 \mathrm{~mL})$ se adicionó a un tubo de ensayo que contuvo un volumen también determinado $(2 \mathrm{~mL})$ de la sustancia a emulsificar (crudo de petróleo). Posteriormente el contenido de los tubos fue agitado con la ayuda de un vórtex por 2 min y luego puesto en reposo por 24 horas. Finalmente, el índice de emulsificación (E24) se obtuvo de la división del tamaño en milímetros de la emulsión generada, entre el total de la altura en milímetros de la mezcla multiplicada por 100. Los ensayos de E24 fueron probados también empleando diesel, gasolina de 84 octanos y kerosene.

\section{Evaluación de las condiciones del medio en la actividad emulsificante}

Se repitió el ensayo anterior con concentraciones crecientes del ramnolípido desde $0,01 \%$ a $1 \%(\mathrm{p} / \mathrm{v})$. Una vez determinada la concentración que generó el mayor valor tanto de actividad emulsificante como del índice E24, frente al crudo de petróleo, se repitió el ensayo esta vez con diferentes valores del $\mathrm{pH}$, con la finalidad de ver el efecto de tal variación en la actividad emulsificante del ramnolípido a la concentración anteriormente elegida; los valores de $\mathrm{pH}$ a probar fueron: $3,5,7,9$ y 11 . 
De igual forma, cada una de las pruebas anteriormente descritas se repitió para cada uno de los derivados del petróleo a emulsificar: petróleo diesel 2, gasolina de 84 octanos y kerosene.

Enfrentamiento del ramnolípido con los metales pesados

Para el enfrentamiento entre estas dos sustancias, se utilizó concentraciones de 1000 ppm de plomo y 500 ppm de cadmio, en la forma de $\mathrm{Pb}\left(\mathrm{NO}_{3}\right)_{2}$ y $3\left(\mathrm{CdSO}_{4}\right) .8 \mathrm{H}_{2} \mathrm{O}$ disueltas en agua bidestilada, y a una concentración del $0,1 \%(\mathrm{p} / \mathrm{v})$ del ramnolípido para cada caso. Posteriormente, dicha mezcla fue agitada durante $60 \mathrm{~min}$ a $120 \mathrm{rpm}$ a temperatura ambiental para permitir la interacción entre las moléculas del ramnolípido y los iones metálicos. Luego del período de agitación, la mezcla fue filtrada a través de membranas de acetato de celulosa de $0,2 \mu \mathrm{m}$, luego de lo cual cada muestra fue enviada a analizar por espectroscopía de absorción atómica, para determinar la concentración final del metal removido en cada muestra.

\section{Evaluación de las condiciones del medio en la captación del metal}

El ensayo fue nuevamente realizado esta vez con concentraciones del ramnolípido del 0,01\%; se realizó variaciones en el $\mathrm{pH}$ del medio con la finalidad de ver el efecto de tal variante en la tasa de remoción de metales con la concentración del biosurfactante anteriormente elegida; los valores de $\mathrm{pH}$ a probar fueron: 3, 5, 7,9 y 11 .

\section{Análisis de datos}

Todos los ensayos fueron realizados por triplicado para poder presentar valores promedio resultantes por cada experiencia realizada. Los datos obtenidos se analizaron con el uso del software estadístico SPSS 17,0.

\section{RESULTADOS Y DISCUSIÓN}

\section{Selección de la cepa bacteriana}

Las cepas bacterianas elegidas para evaluar su actividad emulsificante presentaron crecimiento en el medio Millis suplementado con crudo de petróleo. Asimismo se evaluó la actividad emulsificante de cada una de las cepas frente a crudo de petróleo, obteniéndose los siguientes resultados ( tabla 1 ):

Tabla 1. Cepas bacterianas seleccionadas para el estudio

\begin{tabular}{ccc}
\hline Cepa bacteriana & $\begin{array}{c}\text { Código de } \\
\text { identificación }\end{array}$ & $\begin{array}{c}\text { Actividad } \\
\text { emulsificante } \\
\text { (UAE/mL) }\end{array}$ \\
Pseudomonas fluorescens & MP & - \\
Pseudomonas aeruginosa & 66 & 0,2071 \\
Pseudomonas aeruginosa & 25 & 0,5343 \\
Pseudomonas aeruginosa & 74 & 0,1752 \\
Pseudomonas aeruginosa & AP & - \\
\hline
\end{tabular}

De acuerdo a los resultados obtenidos, se decidió utilizar la cepa Pseudomonas aeruginosa 25 para el resto del estudio, recodificándola como Pseudomonas aeruginosa PB 25. 


\section{Producción del ramnolípido}

Luego de un periodo de incubación de 120 horas en condiciones estáticas a una temperatura ambiental promedio de $25^{\circ} \mathrm{C}$, se obtuvo la curva de crecimiento correspondiente en la cual se refleja la cinética de producción de ramnolípidos y el consumo de glicerol (figura 2). Se logró determinar una velocidad específica de crecimiento $(\mu)$ de $0,0285 \mathrm{~h}^{-1}$ y un tiempo de duplicación $\left(\mathrm{t}_{\mathrm{d}}\right.$ ) equivalente a 24,321 horas. La concentración máxima de ramnolípidos en el medio de cultivo fue de $2,47 \mathrm{~g} / \mathrm{L}$ correspondientes a un rendimiento total $\left(\mathrm{Y}_{\mathrm{x} / \mathrm{s}}\right)$ de 0,13 gramos de ramnolípido por gramo de glicerol y a una productividad volumétrica (Q) de 0,082 gramos de ramnolípido por hora y por litro del medio de cultivo.

La producción de biosurfactantes es un rasgo característico que se obtiene sólo bajo ciertas condiciones que dependen, principalmente, de factores tanto nutricionales como ambientales. De esta forma, si bien los valores obtenidos con respecto a la velocidad específica de crecimiento $(\mu)$ y el tiempo generacional $\left(\mathrm{t}_{\mathrm{g}}\right)$ son muy bajos en comparación a los valores usuales para cualquier microorganismo y a los obtenidos por otros autores ${ }^{6,4}$, hay que tomar en cuenta que este hecho se debe principalmente a la diferencia en cuanto a las condiciones de incubación y, concretamente, al hecho de la falta de agitación.

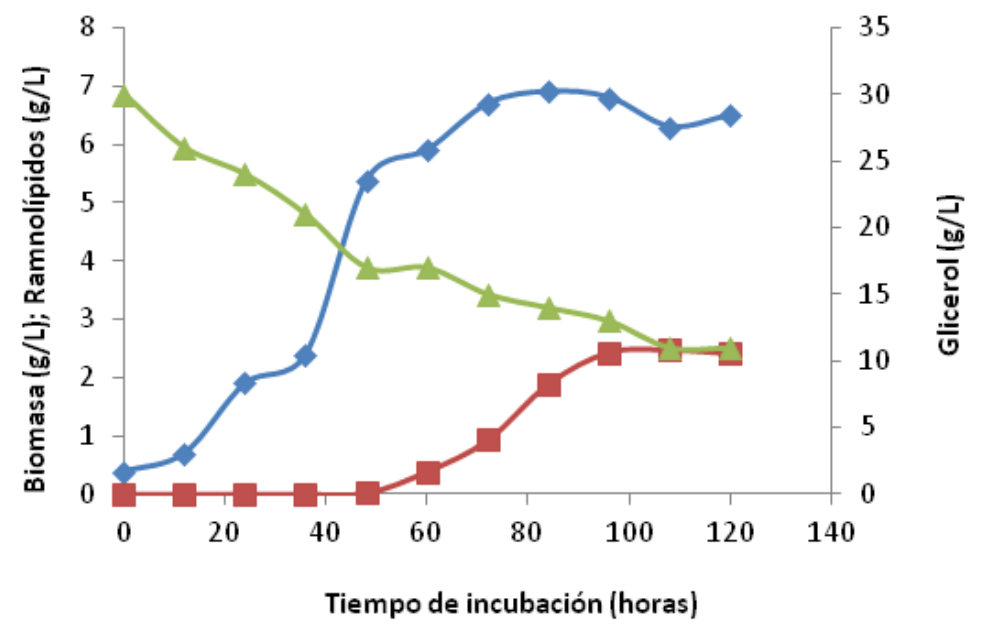

Figura 2. Patrones de crecimiento microbiano de Pseudomonas aeruginosa PB25 ( $\bullet$ ),

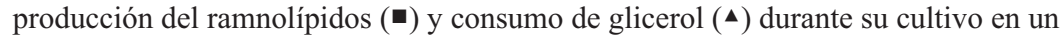
medio mínimo mineral suplementado con $30 \mathrm{~g} / \mathrm{L}$ de glicerol

Los niveles de producción de los ramnolípidos de la cepa en estudio, son bastante acordes e, incluso, superiores a los obtenidos por otros autores que emplearon una agitación constante durante el cultivo del mismo género microbiano ${ }^{4,9,23}$.

\section{Evaluación de la actividad emulsificante}

Puede observarse valores máximos de actividad emulsificante de 3,003 UAE/mL y del índice de emulsificación E24 de $47 \%$ para el uso de concentraciones del 1\% (p/v) del biosurfactante sobre petróleo crudo, motivo por el cual se eligió esta concentración para el resto de pruebas ( tabla 2). 
Tabla 2. Efecto de la concentración los ramnolípidos producidos por Pseudomonas aeruginosa $\mathrm{PB} 25$ sobre la Actividad emulsificante (UAE/mL) y el índice de emulsión E24 (\%) sobre petróleo crudo.

\section{Concentración de ramnolípidos $(\%, p / v)$}

0,01

0,1

1

\section{Actividad emulsificante}

(UAE / mL)

0,907

1,470

3,003
Índice de emulsificación E24

(\%)

15

40

47

Asimismo, es posible observar que el aumento del valor de $\mathrm{pH}$ del medio generó igualmente un aumento en los índices de emulsificación E24 obtenidos frente a los hidrocarburos analizados ( figura 3).

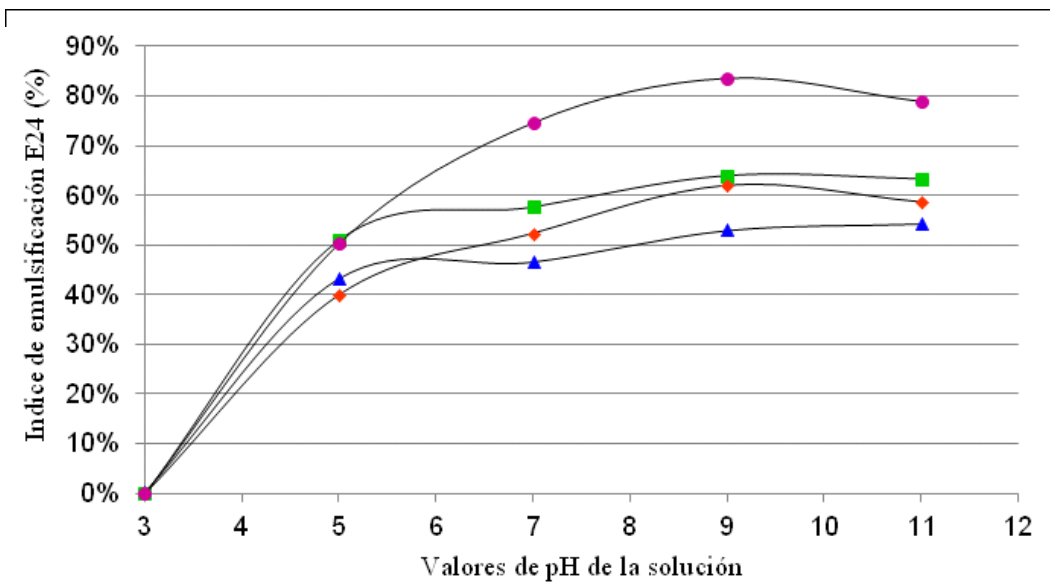

Figura 3. Efecto del pH sobre el índice de emulsificación E24 (\%) de ramnolípidos ( 1\%) producidos por Pseudomonas aeruginosa PB25, sobre petróleo crudo (४), petróleo diesel 2

$(\bullet)$, gasolina de 84 octanos $(\bullet)$ y kerosene $(\bullet)$

Con respecto a la actividad emulsificante de los ramnolípidos frente a los hidrocarburos empleados y su capacidad de unión a los iones de metales pesados, debe señalarse que estas dos propiedades del compuesto microbiano se ven muy influenciadas, principalmente por dos factores: la concentración de surfactante en el medio y el valor del pH. La concentración del surfactante se relaciona directamente con la formación de micelas en el medio lo cual facilita los fenómenos de emulsificación y de unión a iones metálicos, pero más importante aún, el efecto generado por el $\mathrm{pH}$ del medio parece ejercer un papel mucho más importante, ligado al grado de ionización de la porción carboxílica de la molécula del ramnolípido ${ }^{4}$. El incremento 
del $\mathrm{pH}$ favorece la ionización de la porción carboxílica de la molécula del ramnolípido (RCOO') en la solución. Esto, a su vez, mejora su solubilidad en el agua incrementando la actividad emulsificante y brindando más sitios de unión para los cationes metálicos sobre el biosurfactante, conduciendo a un incremento en la solubilidad de los hidrocarburos y de los metales que a su vez quedan atrapados en el interior de sus micelas ${ }^{1}$.

\section{Evaluación de la unión a metales pesados}

Los resultados de la pruebas para la evaluación de la capacidad de remoción de metales pesados en solución, muestran que valores elevados se obtienen con el uso de concentraciones del $0,1 \% \mathrm{p} / \mathrm{v}$ ( tabla 3), motivo por el cual se utilizó esta concentración para las pruebas siguientes. Asimismo, es posible observar que las variaciones en el pH de la solución del metal favorecen la capacidad de remoción por parte del ramnolípido, consiguiéndose un valor máximo del 98\% para el plomo a pH 9 y del 99\% para el cadmio a pH 11 (tabla 4).

Tabla 3. Influencia de la concentración de ramnolípidos de Pseudomonas aeruginosa PB25 en el porcentaje de remoción de cadmio y plomo

\begin{tabular}{ccc}
\hline $\begin{array}{c}\text { Concentración de } \\
\text { ramnolípidos }(\mathbf{\%}, \mathbf{p} / \mathbf{v})\end{array}$ & Plomo (1000 ppm) & Cadmio (500 ppm) \\
0,01 & $37 \%$ & $19 \%$ \\
0,1 & $91 \%$ & $75 \%$ \\
\hline
\end{tabular}

Tabla 4. Porcentaje de remoción de plomo y cadmio, por ramnolípidos producidos por Pseudomonas aeruginosa PB25 al 0,1\% p/v , frente a variaciones en el $\mathrm{pH}$ del medio.

\begin{tabular}{l|ccccc}
\hline \multirow{2}{*}{ Metal pesado } & \multicolumn{5}{c}{ pH del medio (ramnolípido al 1\% p/v) } \\
\cline { 2 - 6 } & $\mathbf{3}$ & $\mathbf{5}$ & $\mathbf{7}$ & $\mathbf{9}$ & $\mathbf{1 1}$ \\
\hline Plomo (1000 ppm) & $75 \%$ & $88 \%$ & $91 \%$ & $98 \%$ & $97 \%$ \\
Cadmio (1000 ppm) & $46 \%$ & $64 \%$ & $75 \%$ & $90 \%$ & $99 \%$ \\
\hline
\end{tabular}

La prueba de Shapiro-Wilks comprobó la normalidad de la distribución de los datos obtenidos, al obtener en todos los casos valores por encima del $\mathrm{p}<0,05$, por lo cual se procedió a emplear el prueba de Levene con la cual se comprobó la homogeneidad de las varianzas de los datos obtenidos de remoción de metales por cada $\mathrm{pH}$ analizado; luego se procedió al empleo de la prueba de ANOVA donde se obtuvo un nivel de significancia por debajo del valor asumido $(\mathrm{p}<0,05)$ lo cual confirma que existe diferencia significativa entre los niveles remoción de metales frente a los valores de $\mathrm{pH}$ ensayados, por lo cual se utilizó la prueba de Scheffe para determinar en qué casos se da esta diferencia ( tabla 5). 
Tabla 5. Resultados de la prueba de Scheffe $(\mathrm{p}<0,05)$

\begin{tabular}{c|ccccc}
\hline & \multicolumn{5}{|c}{$\begin{array}{c}\text { Prueba de Scheffe } \\
(\mathrm{J}) \mathrm{pH}\end{array}$} \\
\hline (I) $\mathrm{pH}$ & 3 & 5 & 7 & 9 & 11 \\
3 & &, 000 &, 000 &, 000 &, 000 \\
5 &, 000 & &, 285 &, 001 &, 003 \\
7 &, 000 &, 285 & &, 025 &, 093 \\
9 &, 000 &, 001 &, 025 & &, 915 \\
11 & 000 & 003 & 093 & 915 & \\
\hline
\end{tabular}

(I),(J) son denominaciones que se da a los $\mathrm{pH}$ para poderlos comparar entre ellos mismos y averiguar en qué caso, se da la diferencia significativa en la remoción de metal pesado. 3-todos: diferencia significativa

5-7: no hay diferencias 5-9,11: diferencia significativa

7-9-11: no hay diferencias significativas

Esto significa que da lo mismo usar el ramnolípido en el rango de $\mathrm{pH}$ entre 7 y 11 puesto que va a tener la misma eficacia, pero a valores de $\mathrm{pH}$ menores a 5 se empieza a apreciar una diferencia significativa en la capacidad de remoción.

\section{CONCLUSIONES}

Se comprobó la capacidad de generar emulsiones de hidrocarburos con el uso de soluciones puras del ramnolípido producido por Pseudomonas aeruginosa PB 25.

El ramnolípido producido por $P$. aeruginosa PB25 mostró eficiencia en la remoción de metales pesados como cadmio y plomo.

Las propiedades demostradas por el surfactante descrito, sugieren su empleo en procesos de biorremediación.

\section{AGRADECIMIENTO}

Agradecemos al Programa de Iniciación Científica 2009 y al Fondo de Apoyo de Tesis de la Universidad Nacional Mayor de San Marcos por el apoyo económico para la realización de la investigación.

\section{BIBLIOGRAFÍA}

1. Neilson, J., Artiola, J. y Maier, R. Journal of Environmental Quality. 2003; 32:899 - 908.

2. Mohan, P., Nakhla, G. y Yanful, E. Journal of Environmental Engineering. 2006;132: 279-283.

3. Abalos, A.; Pinaso, A.; Infante, M.R.; Casals, M.; Garcia, F. y Manresa, A.. Langmuir. 2001;17:. 1367-1371.

4. Mulligan, Catherine and Gibbs, Bernard. Proceedings of the Indian National Science Academy. 2004; 70:31 - 55.

5. Toren, A., Navon-Venezia, S., Ron, E. y Rosenberg, E. Applied and Environmental Microbiology. 2001; 67:1102 - 1106. 
6. Zhang, Guo-Liang, Wu, Yue-Ting, Qian, Xin-Ping and Meng, Qin. Journal of Zhejiang University Science. 2005;6B: 725 - 730.

7. Lovaglio,R., Dos Santos, F., Jafelicci, M., y Contiero, J. Colloids and Surfaces B: Biointerfaces. 2011; 85:301-305.

8. Mulligan, C.N., Yong, R.N. and Gibbs, B.F. Engineering Geology. 2001; 60: 371-380.

9. Guerra-Santos, L., Kappeli, O. y Fiechter, A. Applied and environmental microbiology. 1984;48:301-305.

10. Mukherjee, S., Das, P. y Sen, Ramkrishna. Trends in Biotechnology. 2006;24 :509-515.

11. Wang, Q., Fang, X., Bai, B., Liang, X., Shuler, P., Goddard III, W. y Tang, Y. Biotechnology and Bioengineering. 2007; 98 :842-853.

12. Maier, R. y Soberon-Chavez, G. Applied Microbiology and Biotechnology. 2000; 54:625-633

13. Noordman, W.; WatcheR, J.; De Boer, G. and Janssen, D. Journal of Biotechnology. 2002; $94: 195-212$.

14. Harvey, S.; Elashi, I.; Valdés, J.; Kamely, D. and Chakrabarty, A. Biology and Technology.1990; 8: 228-230.

15. Holakoo, L. and Mulligan, C. Presented at Annual Conference of Canadian Society of Civil Engineering, Montreal, Canada, 5-8 June 2002.

16. Mulligan, Catherine and Wang, Suiling. Engineering Geology. 2006; 85:75-81.

17. Navón-Venezia, S., Zosim, Z., Gottlieb, A., Legmann, R., Carmeli, S., Ron, E. y Rosenberg, E. Applied and Environmental Microbiology. 1995;61: 3240-3244.

18. Mulligan, C.N., Yong, R.N. and Gibbs, B.F. Engineering Geology. 2001; 60: 371-380.

19. Ishigami, Y.; Gama, Y.; Hagahara, H.; Motomiya, T. y Yamaguchi, M. Japanese Patent Kokai 63-182,029. 1988.

20. Zhang, Y.y Miller, R. Applied and Environmental Microbiology. 1994; 60:2101 - 2106.

21. Escalante, R. Tesis Grado de Magister en Biotecnología. UNMSM, Fac. Farmacia y Bioquímica, Lima, 2002.

22. Pons, A., Roca, P., Aguilo, C., Garcia, F., Alemany, M. y Palou, A. Journal of Biochemical and Biophysical Methods. 1981; 4:227-231.

23. Wang, Q., Fang, X., Bai, B., Liang, X., Shuler, P., Goddard III, W. and Tang, Y. Biotechnology and Bioengineering. 2007; 98:842 - 853.

24. Abouseoud, M., Maachi, R., Amarane, A., BouderguA, S. y Nabi, A. Desalination. 2008; 223:143-151. 